\title{
AVALIAÇÃO DAS CONSTANTES TÉRMICAS DAS FASES DE DESENVOLVIMENTO DO PSILÍDEO-DE-CONCHA GLYCASPIS BRIMBLECOMBEI EM LABORATÓRIO
}

\author{
Luiz Alexandre Nogueira de Sá - lans@cnpma.embrapa.br \\ Maria Conceição Peres Young Pessoa - young@cnpma.embrapa.br \\ Gabriele Luciana Saqui - pesquisadores da Embrapa Meio Ambiente, C. Postal 69, 13820-000 Jaguariúna-SP - \\ gabysaqui@yahoo.com.br \\ Artur Batista Rocha - artur_ufscar@yahoo.com.br, bolsistas CNPq/PIBIC e IPEF/ESALq/USP, \\ respectivamente, da Embrapa Meio Ambiente Jaguariúna - SP
}

\section{RESUMO}

A influência da temperatura no desenvolvimento dos insetos é fundamental para a tomada de decisão relacionada à estratégia de controle biológico de pragas. Este trabalho teve por objetivo avaliar a necessidades de graus-dias acumulados entre a oviposição e a eclosão de adultos do psilídeo-de-concha Glycaspis brimblecombei (Hemiptera: Psillydae) em resposta às temperaturas máxima e mínima diárias, observadas em condições de sala de criação do Laboratório de Quarentena "Costa Lima" da Embrapa Meio Ambiente, em Jaguariúna-SP. Três gaiolas com a mesma quantidade inicial de adultos (preservadas a razão fêmea: macho de 1,06) foram monitoradas até o aparecimento da primeira geração e mortalidade total dos adultos introduzidos. Durante todo o período foram registradas as temperaturas máxima e mínima $\left(\mathrm{em}^{\circ} \mathrm{C}\right)$ e a umidade relativa $(\mathrm{em} \%)$ diárias. Posteriormente foram realizados os cálculos dos graus-dias pelo método da senóide horizontal, considerando a temperatura base de desenvolvimento do inseto de $9,26^{\circ} \mathrm{C}$. Os cálculos foram realizados utilizando programa eletrônico da Universidade da Califórnia - Davis (UC-IPM). Como resultado, obteve-se uma necessidade média de 377,49 $\pm 16,09$ GD acumulados entre a oviposição e a eclosão de adultos do inseto, bem como de 350,40 18,62 GD para atingir a morte total de todos os adultos introduzidos.

Palavras-chave: graus-dia, praga florestal, eucalipto, Hemiptera.

\section{THERMAL-CONSTANT EVALUATION OF RED-GUM LERP PSYLLID GLYCASPIS BRIMBLECOMBEI AT LABORATORY CONDITIONS}

\begin{abstract}
The comprehension of the temperature is fundamental toward the correct decision making related to biological control strategies. The present work has as objective to evaluate the degree-day (DD) accumulation necessities during the period of oviposition and adult appearances of red-gum lerp psyllid Glycaspis brimblecombei (Hemiptera: Psillydae), observed considering slave-cages conditions at "Costa Lima" Brazilian National Quarantine Laboratory at Embrapa Environment Jaguariúna City - State of São Paulo, Brazil. Three wood-cages containing the same adult quantities inside (preserved the ratio female: male of 1.06) were monitored until the adults of the first generation appearances. During all the period, maximum and minimum daily temperatures $\left({ }^{\circ} \mathrm{C}\right)$ and moisture (\%) were registered. After that, the degree-day accumulation of each cage was calculated using a sine-horizontal method considering the lower-threshold insect temperature of $9.36{ }^{\circ} \mathrm{C}$. It was also used the USA- University of California- Davis UC-IPM on line program, specific to do this calculus. It was observed as result that it is necessary 377.49 $\pm 16.09 \mathrm{DD}$ to oviposition - adult
\end{abstract}


appearance period, as well as, $350.40 \pm 18.6$ DD to reach the total mortality of introduced adults.

Key-words: degree-day, thermal constant, forest pest, Eucaliptus, Hemiptera.

\section{INTRODUÇÃO}

Vários fatores abióticos influenciam o tempo de desenvolvimento dos insetos, tais como a umidade relativa, temperatura, luz, entre outros. Entretanto, por serem poiquilotérmicos, e assim manterem a temperatura do corpo próxima àquela do ambiente, a temperatura torna-se o principal fator de influência direta e indiretamente sobre seu desenvolvimento e comportamento. Por essa razão a compreensão da influência desse fator no tempo de seu desenvolvimento é fundamental para a tomada de decisão relacionada à estratégia de controle biológico de pragas.

A temperatura representa, portanto, uma das formas de energia (calor) que pode ser acumulada diariamente para assegurar a sobrevivência e o desenvolvimento do ciclo de vida do inseto; bem como de outras atividades.

Cada inseto possui, um valor específico de temperatura que reflete um limitante inferior de energia necessária para assegurar a manutenção das suas condições mínimas vitais diárias (ou seja, sua temperatura limiar de desenvolvimento). Acima deste valor, a energia diária obtida, em unidade de graus-dia (também conhecido como unidades de calor) diária, é armazenada pelo inseto para a realização de suas atividades (inclusive de desenvolvimento e reprodução). Desse modo, conhecendo-se o limiar de desenvolvimento do inseto e as temperaturas máxima e mínima diárias registradas durante o período de cada fase de seu desenvolvimento, permite-se estimar a energia acumulada em unidades de graus-dias necessária para seu ciclo.

A obtenção do ciclo de desenvolvimento do inseto determinado pelo seu fator de maior influência (temperatura) possibilita refletir seu tempo de desenvolvimento em função de particularidades locais do ambiente onde se encontra. Assim, quando apresentado o ciclo de desenvolvimento do inseto em grausdias ele torna-se geral, uma vez que se estende automaticamente quando o inseto é observado em locais de temperaturas menores, bem como diminui quando encontrado em regiões de maiores temperaturas. Por mostrar como o calor diário fornecido pelo sol influencia o desenvolvimento do inseto (e também aplicável ao desenvolvimento das culturas), o cálculo de graus-dias torna-se uma ferramenta de previsão do crescimento do inseto bem mais ampla na estimativa do comportamento dos insetos e na seleção de variáveis mais bem adaptadas ao ambiente, sendo portanto de fundamental importância para o controle biológico de pragas no contexto de programas específicos de manejo integrado de pragas.

Prues (1983) e, recentemente, Fraisse et al. (2007) disponibilizaram informações sobre os vários métodos de cálculo de grausdias disponíveis. Entre eles citam-se os métodos: a) padrão (ou retângulo); b) crescimento de graus-dia modificado; c) curva-senóide; d) integração média horária; etc. Apesar dos cálculos necessários, é cada vez mais freqüente a disponibilização de ferramentas computacionais que auxiliem a agilização da obtenção dos valores de graus-dias, tais como aquelas fornecidas pelo AgClimate Web site da Universidade da Flórida, citado por Fraisse et al. (2007), e o programa eletrônico "IPM: Degree-Day Calculator" disponibilizado pela Universidade da Califórnia - Davis (University of California- Davis. UC-IPM online, 2008).

Vários trabalhos no Brasil também vêm apontando os benefícios da definição de ciclos de desenvolvimento de insetos-praga e de culturas agrícolas nacionais em graus-dias, inseridos no contexto do manejo integrado de pragas ou de programas de prevenção de pragas e doenças (Pessoa, 1994; Marur \& Ruano, 
2001; Mendes et al., 2005; Naves \& Souza, 2009; entre outros).

Com particular atenção para o psilídeo-de-concha Glycaspis brimblecombei, conhecida praga do eucalipto, a definição de estratégias voltadas ao seu controle biológico pode ser mais bem conduzida se definidos seu comportamento nas condições das florestas nacionais em termos de graus-dias. Os trabalhos de monitoramento da praga em diferentes estados brasileiros por Sá et al. (2005), Couto et al. (2005), Dal Pogetto et al. (2005) e Lima et al. (2005), foram suficientes para delinear aspectos importantes de comportamento dos insetos, e justificando as recomendações de manejo atualmente em vigor. A praga por já estar estabelecida no ambiente florestal brasileiro (Sá \& Wilcken, 2004a,b; Sá, 2004) e, portanto, é considerada praga de importância econômica nos hortos florestais de eucalipto, e não mais praga de importância quarentenária (A1). Assim, é encontrada em diferentes regiões com suas particularidades climáticas especificas, conferindo sérios problemas ao setor florestal nacional; e exigindo um avanço contínuo na obtenção de informações que favoreçam especificar estratégias mais adequadas aos programas de manejo integrado de pragas localmente.

Este trabalho apresenta considerações preliminares sobre o desenvolvimento do psilídeo-de-concha em graus-dias acumulados, atribuindo a importância do fator temperatura aos resultados obtidos.

\section{MATERIAL E MÉTODOS}

\section{A) Condições Laboratoriais Avaliadas}

Os dados experimentais resultantes do monitoramento de três gaiolas de criação de G. brimblecombei, identificadas por GC10, GC22 e GC24, mantidas em sala de criação de insetos localizada no Laboratório de Quarentena "Costa Lima" da Embrapa
Meio Ambiente foram utilizados neste trabalho (Saqui et al., 2009a,b,c). As gaiolas foram confeccionadas em madeira $(43,5 \times 40$ x $80 \mathrm{~cm}^{3}$ ) e em seu interior disponibilizados quinze tubetes plantados com Eucaliptus camaldulensis, preferencial para a infestação do inseto em campo. Posteriormente, foram dispostas em sala de criação de insetos mantendo-se a temperatura a $25 \pm 2{ }^{\circ} \mathrm{C}$, UR $60 \pm 10 \%$ e fotofase de $12 \mathrm{~h}$. A mesma quantidade de adultos foi introduzida em todas as gaiolas, a saber, de 35 fêmeas e 33 machos, mantendo a proporção de fêmeas: machos de 1,06. As infestações das gaiolas ocorreram da seguinte forma: gaiola $\mathrm{GC} 10 \mathrm{em}$ 22/10/08; gaiola GC22 em 27/10/08; e gaiola GC24 em 20/10/08. As três gaiolas foram monitoradas quanto às quantidades diárias de machos e de fêmeas (vivos e mortos) em seu interior, identificando-se também as respectivas datas de aparecimento da primeira geração e da mortalidade total dos adultos introduzidos. As temperaturas máxima e mínima $\left(\mathrm{em}^{\circ} \mathrm{C}\right)$ e as umidades relativa máxima e mínima (em \%) das gaiolas também foram monitoradas diariamente durante todo o período. Os resultados obtidos destes monitoramentos foram disponibilizados de forma detalhada em Saqui et al. (2009 a,b,c). Para efeito deste trabalho, foram consideradas as seguintes informações sobre morte total de adultos e aparecimento da primeira geração dos adultos introduzidos, resultantes desses monitoramentos, a saber:

a) GC10 - Morte total de adultos em 12/11/08 e aparecimento da $1^{\mathrm{a}}$ geração proveniente dos adultos introduzidos em 13/11/08;

b) GC22 - Morte total de adultos em 17/11/08 e aparecimento da $1^{\mathrm{a}}$ geração proveniente dos adultos introduzidos em 19/11/08;

c) GC24 - Morte total de adultos em 08/11/08 e aparecimento da $1^{\mathrm{a}}$ geração proveniente dos adultos introduzidos em 10/11/08. 
Também as informações de temperaturas, máxima e mínima, diárias observadas desde a introdução dos adultos até o aparecimento da primeira geração, nas gaiolas GC10, GC22 e GC24 (Saqui et al., 2009 a,b,c), foram utilizadas como entrada de dados do programa de cálculo de graus-dias diário, o qual será mais bem descrito posteriormente. As médias das temperaturas e umidades relativas, máximas e mínimas, são apresentadas na Tabela 1; nela também se observa a média geral das temperaturas e umidades relativas.

Tabela 1. Médias registradas para temperaturas e umidades relativa, máximas e mínimas, observadas da introdução de adultos até o aparecimento da primeira geração, nas gaiolas GC10, GC22 e GC24 (Fonte dos dados: Adaptados de Saqui et al., 2009 a,b,c).

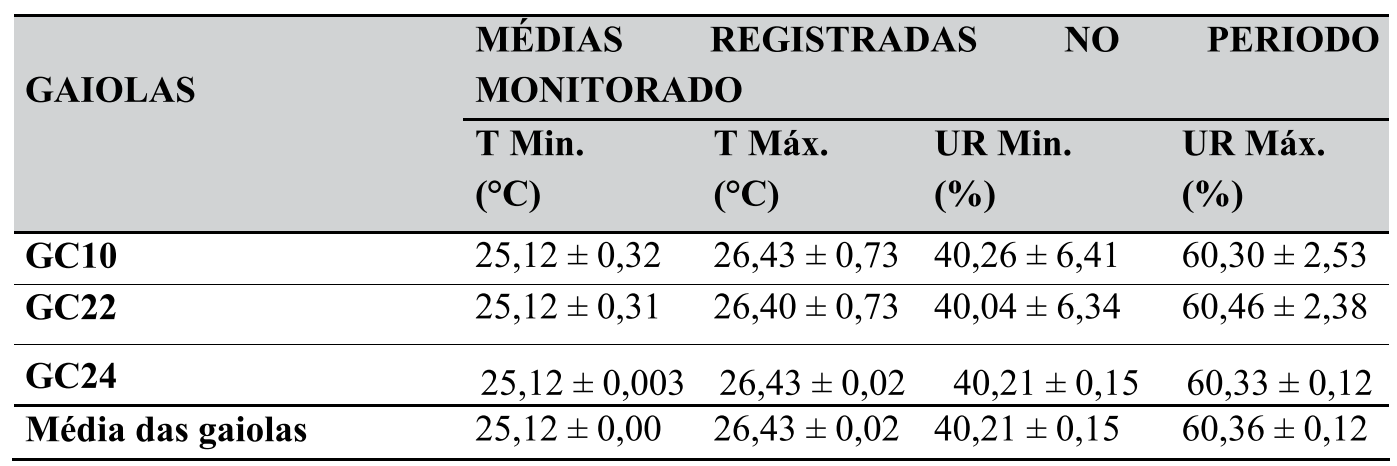

\section{B) Método de Cálculo da Constante Térmi- ca de Desenvolvimento}

A realização dos cálculos dos grausdias diários, bem como acumulados durante o período de desenvolvimento dos insetos presentes em cada gaiola, foi realizado utilizando programa eletrônico disponibilizado pela Universidade da Califórnia - Davis (University of Califórnia- Davis. UC-IPM online, 2008).

O método curva-senóide (ou método de Baskerville-Emin (BE), ou curva-senoidal, curva-seno como também conhecido) de cálculo de graus-dias utiliza uma curva senóide ajustada ao perfil das temperaturas, máxima e mínima. Assim, calcula a área abaixo da curva e acima do limiar de desenvolvimento apresentando esta estimativa para o grau-dia diário. Para um conjunto pequeno de dados de entrada, este método de cálculo se aproxima do método do retângulo. Para períodos maiores, o método curva-senóide apresenta uma melhor estimativa para os resultados de graus-dias que o anteriormente citado (Fraisse et al., 2007).

Fazendo uso do programa, foram ava- liadas, separadamente, cada uma das três gaiolas estudadas, mediante a inserção das suas respectivas informações de temperaturas máxima e mínima diárias. A temperatura representativa do limiar inferior de desenvolvimento do inseto considerada nos cálculos foi a de $9,36{ }^{\circ} \mathrm{C}$, média para a ordem Hemiptera apresentada por (Nietschke et al., 2007). Este valor foi utilizado em função da ausência desta informação para o psilídeo-de-concha em literatura internacional.

\section{RESULTADOS E DISCUSSÃO}

O método curva-senóide foi utilizado no cálculo de graus-dias e aproximou-se do método do retângulo em função do pequeno período de observação. Os resultados obtidos para cada gaiola avaliada são apresentados na Tabela 2.

Utilizando as informações de grausdias da Tabela 2, as quantidades necessárias de graus-dias acumulados para a ocorrência de morte total dos adultos introduzidos e para o período da oviposição até emergência dos primeiros adultos são apresentadas na Tabela 3 . 
Tabela 2. Graus-dias obtidos utilizando o programa UC-IPM online.

\begin{tabular}{|c|c|c|c|c|c|c|}
\hline \multirow[b]{3}{*}{ Data } & \multicolumn{2}{|l|}{ GC10 } & \multirow[t]{2}{*}{ GC22 } & \multicolumn{3}{|c|}{ GC24 } \\
\hline & & GD_acu & & GD_acu & & GD_acu \\
\hline & GD_dia & mulado & GD_dia & mulado & GD_dia & mulado \\
\hline $20 / 10 / 2008$ & & & & & 16,24 & 16,24 \\
\hline $21 / 10 / 2008$ & & & & & 16,44 & 32,68 \\
\hline $22 / 10 / 2008$ & 16,24 & 16,24 & & & 16,24 & 48,92 \\
\hline $23 / 10 / 2008$ & 16,19 & 32,43 & & & 16,49 & 65,41 \\
\hline $24 / 10 / 2008$ & 16,34 & 48,77 & & & 16,34 & 81,75 \\
\hline $25 / 10 / 2008$ & 16,34 & 65,11 & & & 16,34 & 98,09 \\
\hline $26 / 10 / 2008$ & 16,34 & 81,45 & & & 16,34 & 114,43 \\
\hline $27 / 10 / 2008$ & 17,79 & 99,24 & 17,79 & 17,79 & 17,79 & 132,22 \\
\hline $28 / 10 / 2008$ & 16,39 & 115,63 & 16,39 & 34,18 & 16,39 & 148,61 \\
\hline $29 / 10 / 2008$ & 16,39 & 132,02 & 16,39 & 50,57 & 16,39 & 165 \\
\hline $30 / 10 / 2008$ & 16,39 & 148,41 & 16,39 & 66,96 & 16,39 & 181,39 \\
\hline $31 / 10 / 2008$ & 16,39 & 164,8 & 16,39 & 83,35 & 16,39 & 197,78 \\
\hline $01 / 11 / 2008$ & 16,49 & 181,29 & 16,49 & 99,84 & 16,49 & 214,27 \\
\hline $02 / 11 / 2008$ & 16,49 & 197,78 & 16,49 & 116,33 & 16,49 & 230,76 \\
\hline $03 / 11 / 2008$ & 16,49 & 214,27 & 16,49 & 132,82 & 16,49 & 247,25 \\
\hline $04 / 11 / 2008$ & 16,49 & 230,76 & 16,49 & 149,31 & 16,49 & 263,74 \\
\hline $05 / 11 / 2008$ & 16,49 & 247,25 & 16,49 & 165,8 & 16,49 & 280,23 \\
\hline $06 / 11 / 2008$ & 16,49 & 263,74 & 16,49 & 182,29 & 16,49 & 296,72 \\
\hline $07 / 11 / 2008$ & 16,24 & 279,98 & 16,24 & 198,53 & 16,24 & 312,96 \\
\hline $08 / 11 / 2008$ & 16,24 & 296,22 & 16,24 & 214,77 & 16,24 & 329,2 \\
\hline $09 / 11 / 2008$ & 16,24 & 312,46 & 16,24 & 231,01 & 16,24 & 345,44 \\
\hline $10 / 11 / 2008$ & 16,24 & 328,7 & 16,24 & 247,25 & 16,24 & 361,68 \\
\hline $11 / 11 / 2008$ & 16,39 & 345,09 & 16,39 & 263,64 & & \\
\hline $12 / 11 / 2008$ & 16,34 & 361,43 & 16,34 & 279,98 & & \\
\hline $13 / 11 / 2008$ & 16,09 & $\mathbf{3 7 7 , 5 2}$ & 16,09 & 296,07 & & \\
\hline $14 / 11 / 2008$ & & & 16,09 & 312,16 & & \\
\hline $15 / 11 / 2008$ & & & 16,24 & 328,4 & & \\
\hline $16 / 11 / 2008$ & & & 16,24 & 344,64 & & \\
\hline $17 / 11 / 2008$ & & & 16,24 & 360,88 & & \\
\hline $18 / 11 / 2008$ & & & 16,34 & 377,22 & & \\
\hline $19 / 11 / 2008$ & & & 16,34 & 393,56 & & \\
\hline
\end{tabular}

Tabela 3. Graus-dias acumulados para a ocorrência de morte total dos adultos introduzidos e para o período da oviposição até emergência dos primeiros adultos.

\begin{tabular}{lcc}
\hline & $\begin{array}{l}\text { GD } \\
\text { Morte total dos } \\
\text { introduzidos }\end{array}$ & $\begin{array}{l}\text { GD } \\
\text { Oviposição até emergência dos } \\
\text { primeiros adultos }\end{array}$ \\
\hline GC10 & 361,43 & 377,52 \\
GC22 & 360,88 & 393,56 \\
GC24 & 328,90 & 361,38 \\
\hline Média & $350,40 \pm 18,62$ & $377,49 \pm 16,09$ \\
\hline
\end{tabular}


A média obtida a partir dos resultados observados para cada gaiola, evidenciou a necessidade $377,49 \pm 16,09$ GD acumulados para o ciclo de desenvolvimento do inseto (aqui considerado da oviposição até a emergência dos primeiros adultos). Este valor está muito próximo do apontado por Nietschke et al. (2007), que indicaram para a ordem Hemiptera a média de 363,4 GD para o mesmo período.

Observou-se também a necessidade média de 350,40 $\pm 18,62$ GD para a ocorrência de mortalidade total de todos os adultos introduzidos nas gaiolas. Convém não confundir esta mortalidade com a longevidade de adultos. Na primeira, apresenta-se a necessidade de graus- dias para que todos os insetos adultos introduzidos na gaiola tenham morrido, enquanto a longevidade é a esperança de vida da população dos insetos (ou seja, a expectativa média de vida da população). Em particular, com relação à determinação da longevidade de machos e de fêmeas em condições de sala de criação, estudos conduzidos pelo Laboratório de Quarentena "Costa Lima" mostraram que a relação fêmea: macho, da infestação inicial das gaiolas, vêm justificando a variação observada nas longevidades de machos e de fêmeas, o que estão sendo mais bem investigado.

A repetição de novos experimentos, visando determinar o ciclo de vida do inseto em unidades de graus-dias estão em andamento, uma vez que não se identificaram todas as fases de desenvolvimento do inseto pelo acompanhamento realizado neste trabalho; dada a falta dos registros de observações das primeiras conchas e do acompanhamento do ciclo de vida da primeira geração.

\section{CONCLUSÕES}

Os dados de monitoramento de gaiolas em condições de sala de criação em laboratório mantidas a temperaturas máxima e mínima médias de $26,43 \pm 0,02{ }^{\circ} \mathrm{C}$ e $25,12 \pm 0,00{ }^{\circ} \mathrm{C}$, respectivamente, e umidades relativas máxima e mínima médias de $60,36 \pm 0,12 \%$ e $40,21 \pm$ $0,15{ }^{\circ} \mathrm{C}$, respectivamente, indicaram que foram necessários:

a) $350,40 \pm 18,62 \mathrm{GD}$ para a ocorrência de morte total de todos os adultos do psilídeo introduzidos;

b) $377,49 \pm 16,09 \mathrm{GD}$ para se completar o período da oviposição até a emergência dos primeiros adultos;

Existe a necessidade de novos estudos visando determinar o ciclo de vida do inseto em necessidades de graus-dia.

\section{REFERÊNCIAS BIBLIOGRÁFICAS}

COUTO, E. B. do; FERREIRA-FILHO, P. J.; WILCKEN, C. F.; MOURA, M. A.; FERNANDES, B. V.; SÁ, L. A. N. de; MIGRAY, L.; OLIVEIRA, F. H. M. Monitoramento do psilideo-de-concha Glycaspis brimblecombei (HEMIPTERA:PSYLLIDAE) e de seus inimigos naturais em florestas de eucalipto. II. Regiões de Bocaiúva e João Pinheiro, MG. In: SIMPÓSIO DE CONTROLE BIOLÓGICO, 9., 2005, Recife. Anais. Recife: SEB, 2005. p.154.

DAL POGETTO, M. H. F. do A.; LIMA, A. C. V.; FERREIRA FILHO, P. J.; COUTO, E. B. do; SÁ, L. A. N. de; NEVES, E. Monitoramento do psilídeo-de-concha Glycaspis brimblecombei (Hemiptera: Psyliidae) e de seus inimigos naturais em florestas de eucalipto. II. Região de Curvelo, MG, 2004-2005. In: SIICUSP - SIMPÓSIO INTERNACIONAL DE INICIAÇÃO CIENTÍFICA DA UNIVERSIDADE DE SÃO PAULO: Evento Agropecuária, 13., 2005, Piracicaba. Resumos. Disponível em: $<$ http:// www.usp.br/siicusp/13osiicusp/aprovados/ficha5177.htm>. Acesso em: 16 jan. 2006.

FRAISSE, C.W.; BELLOW, J.; BROWN, C. Degree days: heating, cooling, and growing. Gainesville: Agricultural and Biological Engi- 
neering Department, Florida Cooperative Extension Service, Institute of Food and Agricultural Sciences, University of Florida. October, 2007. 7p. (ABE 381).

LIMA, A. C. V.; DAL POGETTO, M. H. F. do A.; COUTO, E. B. do; MOCA, Y.; SÁ, L. A. N. de; FERREIRA FILHO, P. J. Monitoramento do psilídeo-de-concha Glycaspis brimblecombei (Hemiptera: Psyliidae) e de seus inimigos naturais em florestas de eucalipto. I. Região centro-oeste paulista, 2004-2005. In: SIICUSP - SIMPÓSIO INTERNACIONAL DE INICIAÇÃO CIENTÍFICA DA UNIVERSIDADE DE SÃO PAULO: Evento Agropecuária, 13., 2005, Piracicaba. Resumos... Disponível em: $<$ http:// www.usp.br/siicusp/13osiicusp/aprovados/ficha4566.htm>. Acesso em: 16 jan. 2006.

MARUR, C. J.; RUANO, O. A reference system for determination of developmental stages of upland cotton. Revista Brasileira de Oleaginosas e Fibrosas, Campina Grande, v.5, n.2, p.313-317, 2001.

MENDES, S. M.; BUENO, V. H. P.; CARVALHO, L. M. Desenvolvimento e exigências térmicas de Orius insidiosus (Say) (Hemiptera, Anthocoridae). Revista Brasileira de Entomologia, v.49, n.4, p.575-579, 2005 .

NAVES, P.; SOUSA, E. de. Threshold temperatures and degree-day estimates for development of post-dormancy larvae of Monochamus galloprovincialis (Coleoptera: Cerambycidae). Journal of Pest Science, v.82, n. 1, p.1-6, 2009.

NIETSCHKE，B. S.; MAGAREY，R. D.; BORCHERT, D. M.; CALVIN, D. D.; JONES, E. A developmental database to support insect phenology models. Crop Protection, v.26, n.9, p.1444-1448, 2007.

PESSOA, M. C. P. Y. Simulação e inteligência artificial aplicadas ao estudo da dinâ- mica populacional do bicudo do algodoeiro (Anthonomus grandis Boheman) na região de Campinas, SP. 1994. 132p. Tese (Doutorado) - UNICAMP - Faculdade de Engenharia Elétrica, Campinas-SP.

PRUESS, K. P. Day-degree methods for pest management. Environmental Entomology, v.12, n.4, p.613-619, 1983.

SÁ, L.A.N. Alerta Protef - Ocorrência do Psilídeo de concha (Glycaspis brimblecombei) em florestas de eucalipto no Brasil. IPEF on line, 11 de junho de 2004. Disponível em: <www. ipef.esalq.usp.br>.

SÁ, L. A. N. de; WILCKEN, C. F. Nova praga de florestas está atacando eucalipto no país. A Lavoura, Rio de Janeiro, v.107, n.649, p.4445, 2004a

SÁ, L. A. N.; WILCKEN, C. F. Nova praga exótica no ecossistema florestal. Jaguariúna: Embrapa Meio Ambiente, 2004b. 3p. (Embrapa Meio Ambiente. Comunicado Técnico, 18).

SÁ, L. A. N. de; WILCKEN, C. F.; FRANCHIM, T.; STECCA, L. F. F.; ALMEIDA, G. R. de; PEREIRA, R. A. A.; COUTO, E. B. do; TAKAHASHI, S. S.; TEIXEIRA, J. S. Monitoramento do psilideo-de-concha Glycaspis brimblecombei (Hemiptera:Psyllidae), e de seus inimigos naturais em florestas de eucalipto. I - Regiões de Campinas, Rio Claro, Ribeirão Preto e Sul de Minas Gerais. In: SIMPÓSIO DE CONTROLE BIOLÓGICO, 9., 2005, Recife. Anais... Recife: SEB, 2005. p.182.

SAQUI, G. L.; ROCHA, A. B. O.; PESSOA, M. C. P. Y.; SÁ, L.A.N. Relatório de monitoramento populacional de adultos do psilideo-de-concha, Glycaspis brimblecombei, em condição de sala de criação climatizada - GC10 no período de 22/10/2008 
a 12/11/ 2008. Jaguariúna: Laboratório de Quarentena "Costa Lima"/Embrapa Meio Ambiente, 2009a. 14p. (Relatório Técnico LQC, nº68).

SAQUI, G. L.; ROCHA, A. B. O.; PESSOA, M. C. P. Y.; SÁ, L. A. N. Relatório de monitoramento populacional de adultos do psilideo-de-concha, Glycaspis brimblecombei, em condição de sala de criação climatizada - GC22 no período de 27/10/2008 a 19/11/ 2008. Jaguariúna: Laboratório de Quarentena "Costa Lima"/Embrapa Meio Ambiente, 2009b. 13p. (Relatório Técnico LQC, $\left.n^{\circ} 669\right)$.
SAQUI, G. L.; ROCHA, A. B. O.; PESSOA, M. C. P. Y.; SÁ, L. A. N. de Monitoramento populacional de adultos do psilideo-de-concha, Glycaspis brimblecombei, em condição de sala de criação climatizada - GC24 no período de 20/10/2008 a 10/11/ 2008. Jaguariúna: Laboratório de Quarentena "Costa Lima"/Embrapa Meio Ambiente, 2009c. 15p. (Relatório Técnico LQC, $\mathrm{n}^{\circ} 670$ ).

UNIVERSITY OF CALIFORNIA. Run models and calculate degree-days. Davis: UC IPM, 2008. Disponível em: <http://www.ipm. ucdavis.edu/WEATHER/ddretrieve.html>. Acesso em: 02 set. 2008. 